\title{
Pacific
}

Journal of

Mathematics

\section{HOMOGENEOUS LINKS AND THE SEIFERT MATRIX}

\author{
PedRo M. GonZÁLEZ ManchóN
}

Volume 255 No. 2

February 2012 


\title{
HOMOGENEOUS LINKS AND THE SEIFERT MATRIX
}

\author{
PEDRO M. GonZÁleZ MANCHÓN
}

\begin{abstract}
Homogeneous links were introduced by Peter Cromwell, who proved that the projection surface of these links, given by the Seifert algorithm, has minimal genus. Here we provide a different proof, with a geometric rather than combinatorial flavor. To do this, we first show a direct relation between the Seifert matrix and the decomposition into blocks of the Seifert graph. Precisely, we prove that the Seifert matrix can be arranged in a block triangular form, with small boxes in the diagonal corresponding to the blocks of the Seifert graph. Then we prove that the boxes in the diagonal have nonzero determinant, by looking at an explicit matrix of degrees given by the planar structure of the Seifert graph. The paper also contains a complete classification of homogeneous knots of genus one.
\end{abstract}

\section{Introduction}

Throughout this paper, we assume that all links and diagrams are oriented. Let $F$ be a spanning surface for an oriented link $L$, and let $b: F \times[0,1] \rightarrow \mathbb{R}^{3}$ be a regular neighborhood. Identify $F$ with $F \times\{0\}$. The associated Seifert matrix $M=\left(a_{i j}\right)_{1 \leq i, j \leq n}$ of order $n$ is defined by the linking numbers $a_{i j}=\operatorname{lk}\left(a_{i}, a_{j}^{+}\right)$, where the $a_{i}$ are simple closed oriented curves in $F$ whose homology classes form a basis $\mathscr{B}$ of $H_{1}(F)$, and $a_{i}^{+}=b\left(a_{i} \times 1\right)$ is the lifting of $a_{i}$ out of $F$, in $F \times\{1\}$. Then

$$
n=\operatorname{rk} H_{1}(F)=2 g(F)+\mu-1=1-\chi(F),
$$

where $g(F)$ and $\chi(F)$ are the genus and Euler characteristic of $F$, and $\mu$ is the number of components of the link. Homology with coefficients in $\mathbb{Z}$ is assumed throughout the paper.

Let $\nabla_{L}(z)$ and $\Delta_{L}(x)$ be the Conway and Alexander polynomials of $L$, in the variables $z$ and $x$ respectively, as defined in [Cromwell 2004]. Upon the substitution $z=x^{-1}-x$, we have $\nabla_{L}(z)=\Delta_{L}(x)=\operatorname{det}\left(x M-x^{-1} M^{t}\right)$. Therefore the coefficient $c$ of the highest degree term in $\nabla_{L}(z)$ is $(-1)^{n} \operatorname{det} M$ and the degree

Partially supported by Spanish Project MTM2010-19355 and FEDER.

MSC2010: primary 57M25, 57M27; secondary 57M15, 05C50.

Keywords: homogeneous link, projection surface, Seifert graph, Seifert matrix, Conway polynomial, knot genus, blocks of a graph. 
of $\nabla_{L}(z)$ is $n$, whenever det $M$ does not vanish. In general $\operatorname{deg} \nabla_{L}(z) \leq n$, which provides the famous lower bound on the genus, $\operatorname{deg} \nabla_{L}(z)-\mu+1 \leq 2 g(F)$, and in particular it allows us to deduce that $F$ is a minimal genus spanning surface for $L$ if $\operatorname{det} M \neq 0$.

Now suppose that the spanning surface $F$ has been constructed by applying the Seifert algorithm to a diagram $D$ of the link $L$. We briefly summarize the main features of this construction: start with a diagram $D$ in the $x y$-plane. For each Seifert circle $\alpha$ a Seifert disc $a$ is built in the plane $z=k$, if there are exactly $k$ Seifert circles that contain $\alpha$; we say that the height of $a$ is $k$ and write $h(a)=k$. This collection of discs lives in the upper half-space $\mathbb{R}_{+}^{3}$ and they are stacked in such a way that when viewed from above, the boundary of each disc is visible. To complete the projection surface, insert small twisted rectangles (called bands from now on) at the site of each crossing, choosing the half-twist according to the corresponding crossing. Following [Cromwell 2004], we call $F$ a projection surface.

We can now define a graph $G$ contained in $F$ as follows: take a vertex in each Seifert disc of $F$ and, if two discs are joined by a band, join the corresponding vertices by an edge contained in the band. Label the edge with the sign of the associated crossing in the diagram $D$. This graph, called the Seifert graph of $D$, is in fact a planar graph. The rank rk $G$ of $G$, as defined in graph theory, is one minus the number of vertices plus the number of edges. Since $\chi(F)=s(D)-c(D)$, where $s(D)$ is the number of Seifert circles and $c(D)$ is the number of crossings of $D$, it follows that rk $G=\operatorname{rk} H_{1}(F)$.

In general, we can consider the decomposition $G=B_{1} \cup \cdots \cup B_{k}$ of the graph $G$ into its blocks, which are the maximal connected subgraphs without cut vertices. The part of the projection surface (bands and Seifert discs) that corresponds to a block $B_{i}$ is a submanifold of $F$ and will be denoted by $F_{B_{i}}$, or simply $F_{i}$. The graph $G$ is a deformation retract of the surface $F$, taking $F_{i}$ onto $B_{i}$; in particular $H_{1}(F) \cong H_{1}(G)$ taking $H_{1}\left(F_{i}\right)$ onto $H_{1}\left(B_{i}\right)$ and $\operatorname{rk} G=\operatorname{rk} H_{1}(G)$, an equality sometimes taken as a definition. Now, a basis of $H_{1}(G)$, hence a basis $\mathscr{B}$ of $H_{1}(F)$, can be obtained by juxtaposing basis $\mathscr{B}_{i}$ of $H_{1}\left(B_{i}\right)$, since the cycles in $G$ are precisely the cycles of its blocks [Diestel 2005, Lemma 3.1.1]. In particular, the rank of $G$ is the sum of the ranks of its blocks.

Let $M_{i}$, where $i=1, \ldots, k$, be the Seifert matrix defined by any basis $\mathscr{B}_{i}$ of $H_{1}\left(B_{i}\right)$ (hence of $H_{1}\left(F_{i}\right)$ ). Our main result is this:

Theorem 6. Let $D$ be a connected diagram of an oriented link $L$. Let $G$ be the corresponding Seifert graph and $G=B_{1} \cup \cdots \cup B_{k}$ its decomposition into blocks. Then there is an order in the set of blocks of $G$ for which the Seifert matrix for the projection surface is upper block triangular. More precisely, if $M_{i}$ is the Seifert matrix that corresponds to any basis $\mathscr{B}_{i}$ of $H_{1}\left(B_{i}\right), i=1, \ldots, k$, there exists a 
permutation $\sigma \in S_{k}$ such that the Seifert matrix takes on the form

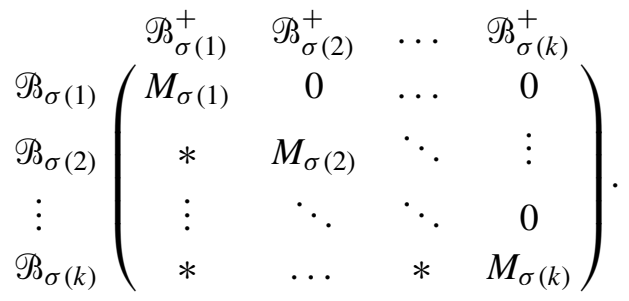

A link is homogeneous if it has a homogeneous diagram, which is a diagram in which all the edges of each block of its Seifert graph have the same sign. Alternating and positive diagrams (links) are homogeneous diagrams (links). The knot $9_{43}$ is an example of a homogeneous link that is neither positive nor alternating. Homogeneous links were introduced in [Cromwell 1989]. In knot theory the adjective homogeneous was first applied to a certain class of braids in [Stallings 1978]. Certainly, the closure of a homogeneous braid is a homogeneous diagram, although there are homogeneous links that cannot be presented as the closure of a homogeneous braid, just as there are alternating links that cannot be presented as the closure of alternating braids. In Cromwell proved the following basic result on homogeneous links:

Theorem [Cromwell 1989; 2004]. Let D be a connected homogeneous diagram of an oriented homogeneous link $L$ and let $G$ be the corresponding Seifert graph. Then the highest degree of $\nabla_{L}(z)$ is the rank of $G$. Let $G=B_{1} \cup \cdots \cup B_{k}$ be the decomposition of $G$ into blocks and $M_{i}, i=1, \ldots, k$, the corresponding Seifert matrices. Then $\operatorname{det} M_{i} \neq 0$ for $i=1, \ldots, k$, and the leading coefficient of $\nabla_{L}(z)$ is

$$
\prod_{i=1}^{k} \epsilon_{i}^{r_{i}}\left|\operatorname{det} M_{i}\right|
$$

where $\epsilon_{i}$ is the sign of the edges in $B_{i}$ and $r_{i}=\operatorname{rk} B_{i}$.

Corollary. A projection surface constructed from a connected homogeneous diagram of an oriented link is a minimal-genus spanning surface for the link.

Cromwell's proof is based on a previous construction of a specific resolving tree for calculating the Conway polynomial [Cromwell 2004, Lemma 7.5.1]. This means that no crossing is switched more than once on any path from the root of the tree to one of its leaves. The skein relation is then considered, at both the level of the diagram and the corresponding Seifert graph, having in mind that to obtain terms involving powers of $z$ when resolving the resolution tree, a crossing must be smoothed in the diagram $D$, or equivalently, an edge must be deleted from the graph $G$. A direct proof of the corollary has been recent and independently 
suggested by M. Hirasawa. The proof, outlined in [Abe 2011] (see also [Ozawa 2011]), is strongly based on a difficult result from [Gabai 1983], which states that the sum of Murasugi of minimal genus surfaces is a minimal-genus surface. Hirasawa applies this result to the portions $F_{i}$ above defined.

In this paper we give a different proof of Cromwell's theorem, based on the close relation between the Seifert matrix and the decomposition into blocks of the Seifert graph stated in Theorem 6. The key point is the understanding of how the parts of the projection surface corresponding to the blocks are geometrically positioned among them. We remark that Theorem 6 can be useful even when the diagram is not homogeneous. A special case, involving fibered knots of genus two formed by plumbing Hopf bands, was already considered in [Melvin and Morton 1986]. We deal with this topic in Section 2.

Since a homogeneous block of the Seifert graph corresponds to an alternating diagram, each little box in the diagonal of the Seifert matrix has nonzero determinant, according to the work by K. Murasugi [1958a; 1958b; 1960] and independently Crowell [1959]. Murasugi's proof was accomplished by working on the Alexander matrix of the Dehn presentation of the link, while Crowell worked with the Wirtinger presentation of the fundamental group. In this paper we will prove this result, the second ingredient of our argument, by looking at an explicit matrix of degrees that uses the planar structure of the Seifert graph (Theorem 9). This will be done in Section 3.

Section 4 contains a complete classification of genus-one homogeneous knots.

\section{An order for the blocks and the Seifert matrix}

The main achievement of this paper is to prove that there is a certain ordered basis of the first homology group of the projection surface for which the Seifert matrix has a block triangular form. We need first to prove that, in a certain sense, there are only two types of blocks, or more precisely, there are only two possible configurations for the portions $F_{B}$ associated to a block $B$.

Let $a, b$ be two Seifert discs. We say that $a$ contains $b$ (written $a \supset b$ ) if the projection onto the $x y$-plane of $a$ contains that of $b$ (see figure). Equivalently, the Seifert circle associated to $a$ contains that associated to $b$, in the $x y$-plane.

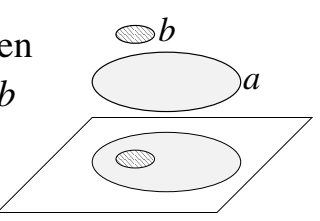

Remark 1. If we project the projection surface onto the $x y$-plane, the only selfintersections of its boundary are given by the crossings of the original diagram $D$, and they are produced by the half-twists of the bands.

In particular the arrangement on the right is not possible. As a result we obtain the next lemma.

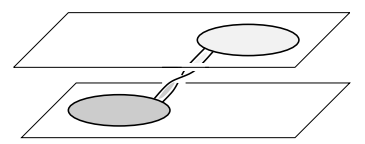


Lemma 2. Let $a, b$ be two Seifert discs connected by a band. Then exactly one of the following three statements holds:

(1) $a \supset b$ and $h(b)=h(a)+1$.

(2) $b \supset a$ and $h(a)=h(b)+1$.

(3) $h(a)=h(b)$.

The proof is easy and left to the reader. Now, we can prove that there are basically two types of blocks. Precisely:

Theorem 3. Let $D$ be a diagram, $F$ its projection surface and $G$ the corresponding Seifert graph. Then all the Seifert discs associated to a block of $G$ have the same height, except possibly one of them which contains all the other, being its height one less.

Proof. Suppose that $a$ and $b$ are two Seifert discs with different height connected by a band, both associated to the same block. By Lemma 2 we may assume that one contains the other; say $a \supset b$. It turns out that there is no other Seifert disc associated to the block with height lower than $b$, since that would make the vertex corresponding to $a$ a cut vertex, according to Remark 1. Analogously, any other disc above $b$ would make (the vertex corresponding to) $b$ a cut vertex.

Hence we have two possible arrangements for (the Seifert discs that correspond to) a block: type I (fried eggs type) and type II (fried eggs with a pan type). In a type II block, the pan is the Seifert disc with lowest height. The two types of blocks are illustrated here:

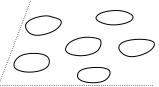

Fried eggs

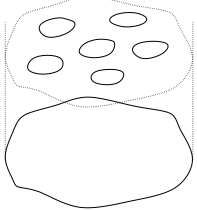

Fried eggs with a pan

Following Cromwell [1989] or Murasugi [1958a; 1958b] we say that a (Seifert) circle is of type I if it does not contain any other circle; otherwise it is of type II. When a type II circle has other circles outside, it is called a decomposing circle. By definition, a special diagram does not contain any decomposing circle. Note that a type II circle is the boundary of the pan of a type II block, assuming that the diagram is connected.

Now, recall from the introduction that the part of the projection surface that corresponds to a block $B_{i}$ is denoted by $F_{i}$, which is a submanifold of $F$. Recall also that, since the cycles of a graph are the cycles of its blocks, we have that a basis of $H_{1}(F)$ can be obtained by juxtaposing a basis for each block. 
Remark 4. Two different $F_{i}$ 's can have at most one common Seifert disc; hence $F$ is the Murasugi sum of the portions $F_{i}$ 's. The proof by Hirasawa mentioned in the introduction follows from this fact.

In order to prove the main theorem, we need the following result of graph theory:

Lemma 5. Let $G$ be a connected finite graph with at least one cut vertex. Then there is a block of $G$ which has exactly one cut vertex of $G$.

Proof. It can be deduced from Proposition 3.1.2 of [Diestel 2005]. It follows a direct argument: delete any cut vertex $v_{0}$ of $C_{0}=G$ and consider $C_{1}=C_{1}^{\prime} \cup\left\{v_{0}\right\}$ where $C_{1}^{\prime}$ is any connected component of $C_{0}-\left\{v_{0}\right\}$. We remark that, under these assumptions, the cut vertices of $C_{1}$ are exactly the cut vertices of $G$ that lie in $C_{1}$, except for $v_{0}$, and that any block of $C_{1}$ is a block of $G$. If $C_{1}$ has no cut vertices, then it is the wanted block. Otherwise we select a cut vertex $v_{1}$ of $C_{1}$ and consider $C_{2}=C_{2}^{\prime} \cup\left\{v_{1}\right\}$ where $C_{2}^{\prime}$ is a connected component of $C_{1}-\left\{v_{1}\right\}$ with $v_{0} \notin C_{2}^{\prime}$. Repeating this process, we finally get a $k \in \mathbb{N}$ such that $C_{k}$ has no cut vertices, hence being the wanted block. Otherwise we would obtain an infinite sequence of distinct vertices $\left\{v_{0}, v_{1}, v_{2}, \ldots\right\}$ in the finite graph $G$, a contradiction.

Theorem 6. Let $D$ be a connected diagram of an oriented link $L$. Let $G$ be the corresponding Seifert graph and $G=B_{1} \cup \cdots \cup B_{k}$ its decomposition into blocks. Then there is an order in the set of blocks of $G$ for which the Seifert matrix for the projection surface is upper block triangular. More precisely, if $M_{i}$ is the Seifert matrix that corresponds to any basis $\mathscr{B}_{i}$ of $H_{1}\left(B_{i}\right), i=1, \ldots, k$, there exists a permutation $\sigma \in S_{k}$ such that the Seifert matrix takes on the form

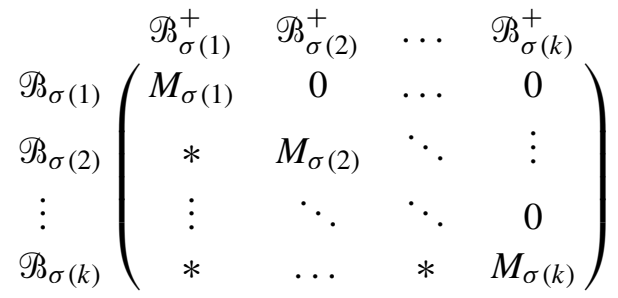

Proof. By Lemma 5, there exists a block $B$ which has exactly one cut vertex. Let $D$ be the Seifert disc associated to the unique cut vertex in $B$. Translated to the surface, this means that the geometric block $F_{B}$ is separated from the rest of the surface $F$, with $D$ as the unique intersection.

We may assume, by induction on the number of blocks, that the Seifert matrix for $F \backslash\left(F_{B} \backslash D\right)$ is upper triangular for a suitable order of the rest of blocks $B_{i}$ 's. Suppose now that the positive orientation of the disc $D$, that looking at $F \times\{1\}$, is upwards. Then, the basis that corresponds to the block $B$ must be added

- at the beginning if $B$ is of type I, or $D$ is an egg of the type II block $B$,

- at the end if $D$ is the pan of the type II block $B$. 
Indeed, if the positive orientation of $D$ were downward, these statements must be interchanged.

In the following displayed figures, the shadowed discs correspond all to the block $B$; the disc $D$, partially shadowed, is part of the two considered blocks, $B$ and any other block $B_{i}$ previously ordered. On $D$ there is an oriented arrow looking upwards, indicating the positive orientation. Suppose now that $g, g_{i} \in$ $H_{1}(F)$ correspond to the blocks $B$ and $B_{i}$ respectively. We have to analyze the three possible cases:

(1) Suppose that $B$ is of type $\mathrm{I}$. We have to see that $\operatorname{lk}\left(g, g_{i}^{+}\right)=0$. This can be easily checked if $B_{i}$ is of type I, or $B_{i}$ is of type II and the disc $D$ is its pan. And it is also true if $B_{i}$ is of type II being $D$ an egg of $B_{i}$, since in this case the eggs would be on different half parts of the pan. To see this, project both blocks $B$ and $B_{i}$ onto the plane $z=h(D)-1$, hence the Seifert discs at height $h(D)$ are now nested inside the pan of the block $B_{i}$ (all the eggs in the same pan). By Remark 1 there is no intersections other than those given by the half-twists of the bands, which means that the two blocks are basically in separated half parts of the pan of $B_{i}$. In particular, a band crossed like this is not possible:

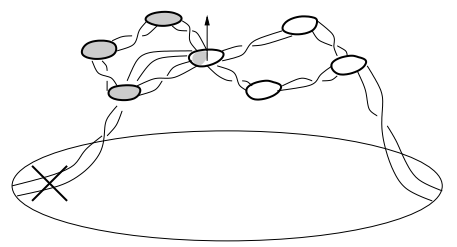

(2) Suppose that $B$ is of type II, and the disc $D$ is an egg of $B$. As in the previous case, we have to see that $\operatorname{lk}\left(g, g_{i}^{+}\right)=0$. This can be easily checked if any other block $B_{i}$ is of type I, or (see figure below) $B_{i}$ is of type II and the disc $D$ is the pan of $B_{i}$.

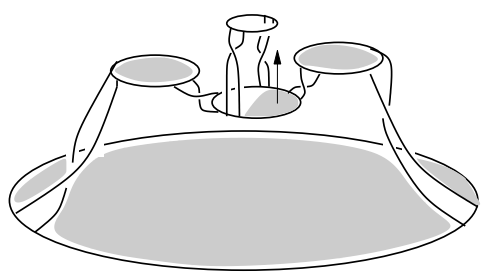

Note that $D$ cannot be an egg of another type II block $B_{i}$. Indeed, if it were, again by Remark 1, the pan would be the same for $B$ and $B_{i}$, hence the blocks $B$ and $B_{i}$ would share at least two vertices. But, by their maximality, different blocks of $G$ overlap in at most one vertex.

(3) Suppose that $B$ is of type II, and the disc $D$ is its pan. In this case we have to see that $\operatorname{lk}\left(g_{i}, g^{+}\right)=0$. This can be easily checked if the block $B_{i}$ is of type I, or 
the disc $D$ is an egg of a type II block $B_{i}$. And it is also true if the disc $D$ is the pan of another type II block $B_{i}$, since in this case, by a similar argument to that used in the first case, the eggs would be on different half parts of the pan, the crossed band shown here not being possible:

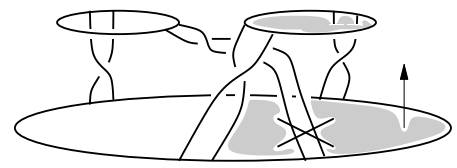

Example 7. Suppose that we wish to find the block triangular form for the Seifert matrix of the link shown here:

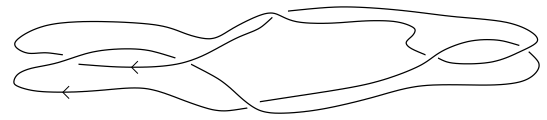

We draw the corresponding Seifert circles and Seifert graph:

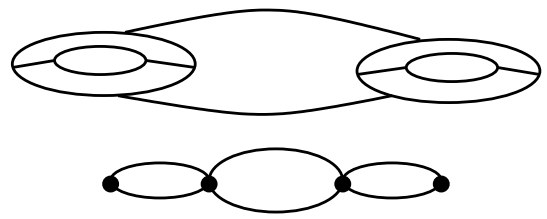

We decompose the Seifert graph into blocks $B_{1}, B_{2}$ and $B_{3}$, from left to right:

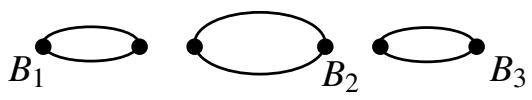

Here is projection surface:

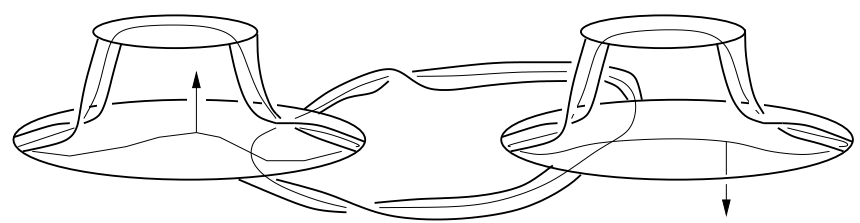

We can consider $B=B_{1}$ as the block with only one cut vertex. Then, if the positive orientation of $D$ is upwards, for the other two blocks the suitable basis is given by the order of blocks $\left\{B_{3}, B_{2}\right\}$, which gives the matrix

$$
\begin{array}{ccc} 
& \mathscr{B}_{3}^{+} & \mathscr{B}_{2}^{+} \\
\mathscr{B}_{3} & * & 0 \\
\mathscr{B}_{2} & * & *
\end{array}
$$

Since $B=B_{1}$ is a block of type II and the disc $D$ that corresponds to the cut vertex is a pan of $B$, according to the proof of Theorem 6 we must add the basis 
for $B$ at the end, obtaining the order $\left\{B_{3}, B_{2}, B_{1}\right\}$ and the matrix

$\begin{array}{cccc} & \mathscr{B}_{3}^{+} & \mathscr{B}_{2}^{+} & \mathscr{B}_{1}^{+} \\ \mathscr{B}_{3} & * & 0 & 0 \\ \mathscr{B}_{2} & * & * & 0 \\ \mathscr{B}_{1} & * & * & *\end{array}$

\section{The box matrix associated to a block}

Recall from the introduction that the coefficient $c$ of the highest degree term in $\nabla_{L}(z)$ is equal to $(-1)^{n} \operatorname{det} M$ and the degree of $\nabla_{L}(z)$ is $n=\operatorname{rk} H_{1}(F)$, whenever $\operatorname{det} M$ does not vanish. By Theorem $6, \operatorname{det} M=\prod_{i=1}^{k} \operatorname{det} M_{i}$ where $M_{i}$ is the Seifert matrix that corresponds to the surface $F_{i}$ associated to the block $B_{i}$ of $G$. Then, in order to prove the theorem stated in the introduction, it is enough to show that, if $B_{i}$ is a block with rank $r_{i}$ and all its edges have sign $\epsilon_{i}$, then the determinant of its Seifert matrix does not vanish and has $\operatorname{sign}\left(-\epsilon_{i}\right)^{r_{i}}$. Indeed, since $n=\operatorname{rk} G$ is the sum of the ranks $r_{i}$ of its blocks, we would have

$$
\begin{aligned}
c & =(-1)^{n} \operatorname{det} M=(-1)^{n} \prod_{i=1}^{k} \operatorname{det} M_{i} \\
& =(-1)^{n} \prod_{i=1}^{k}\left(-\epsilon_{i}\right)^{r_{i}}\left|\operatorname{det} M_{i}\right|=\prod_{i=1}^{k} \epsilon_{i}^{r_{i}}\left|\operatorname{det} M_{i}\right| \neq 0 .
\end{aligned}
$$

Now, the part of the diagram that corresponds to a homogeneous block is alternating (in fact, it is a special alternating diagram), and the result for these links follows from [Murasugi 1960] and [Crowell 1959]. Murasugi's proof was accomplished by working on the Alexander matrix of the Dehn presentation, while Crowell worked with the Wirtinger presentation of the fundamental group of the link. In fact, Crowell's paper rests on a striking application of a graph theoretical result, the Bott-Mayberry matrix tree theorem, an approach also explained in [Burde and Zieschang 2003, Proposition 13.24]. In this section we will prove it (Theorems 9 and 10) by looking at an explicit matrix of degrees defined using the planar structure of the Seifert graph.

Let $D$ be an oriented diagram, $F$ its projection surface and $G$ the corresponding Seifert graph. Let $B$ be a block of $G$. A basis $\left\{g_{1}, \ldots, g_{r}\right\}$ of $H_{1}(B)$ (hence of $H_{1}\left(F_{B}\right)$ ) can be obtained collecting the counterclockwise oriented cycles defined by the boundaries of the bounded regions $R_{i}$ defined by $B$. Let $R_{r+1}$ be the unbounded region defined by this planar graph $B$ (like $R_{5}$ in the figure on the right).

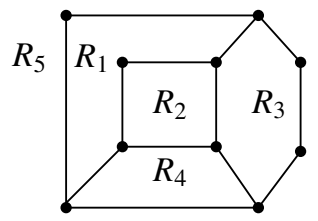


The Seifert graph is a bipartite graph because the projection surface is orientable, hence every circuit in the graph must have an even length. In particular, we can choose a sign for an arbitrary vertex, and extend this labelling to the other vertices in an alternating fashion, when moving along the edges. We also have, for each edge $e$ in $B$, its corresponding sign $\epsilon(e)$ (if the original diagram is homogeneous, this sign is constant in the block). We define $E_{i j}$ as the set of edges in $\partial R_{i} \cap \partial R_{j}$ with the sign arrangement exemplified by the figure. (The edge $e$ belongs to $E_{i j}$ with this arrangement of signs.)

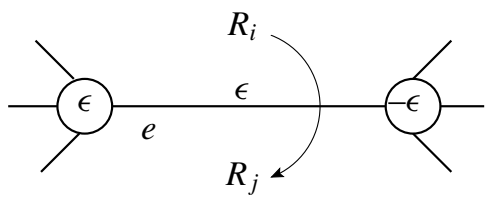

It turns out that $\operatorname{lk}\left(g_{i}, g_{i}^{+}\right)=\frac{1}{2} \sum_{e \in \partial R_{i}}-\epsilon(e)$ and $\operatorname{lk}\left(g_{i}, g_{j}^{+}\right)=\sum_{e \in E_{i j}} \epsilon(e)$. In particular, if the block is homogeneous, let say with sign $\epsilon$, then

$$
\operatorname{lk}\left(g_{i}, g_{i}^{+}\right)=-\epsilon k_{i},
$$

where $2 k_{i}$ is the number of edges in $\partial R_{i}$, and

$$
\operatorname{lk}\left(g_{i}, g_{j}^{+}\right)=\epsilon\left|E_{i j}\right| \text {. }
$$

In other words, $\operatorname{lk}\left(g_{i}, g_{j}^{+}\right)$is the number (with sign $\epsilon$ ) of the edges $e$ in the frontier of the regions $R_{i}$ and $R_{j}$, such that one leaves the $-\epsilon$ signed vertex on the left when going from $R_{i}$ to $R_{j}$ through the edge $e$ (see figure above).

As an example, we display the Seifert matrix associated to the graph of the previous page, assuming that the top left vertex is labelled with sign $\epsilon$; the figure on the right shows the other vertex labels (note that this constitutes a homogeneous block; all the edges have $\operatorname{sign} \epsilon$ ):

$$
\begin{aligned}
& g_{1}^{+} \\
& g_{2} \\
& g_{3} \\
& g_{4}
\end{aligned}\left(\begin{array}{cccc}
-3 \epsilon & g_{2}^{+} & g_{3}^{+} & g_{4}^{+} \\
\epsilon & -2 \epsilon & 0 & 0 \\
0 & \epsilon & -3 \epsilon & 0 \\
\epsilon & 0 & \epsilon & -2 \epsilon
\end{array}\right)
$$

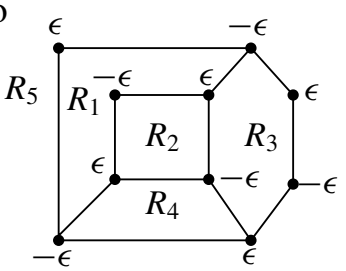

The sets $E_{i j}$ satisfy two properties, which will play later a central role, especially in Theorem 9:

(1) If $e \in \partial R_{i} \cap \partial R_{j}$, then $e \in E_{i j} \Longleftrightarrow e \notin E_{j i}$, and in particular $\left|E_{i j}\right|+\left|E_{j i}\right|$ is the cardinal of the edges in $\partial R_{i} \cap \partial R_{j}$. 
(2) Consider two consecutive edges $e$ and $f$ in the boundary of a certain region $R_{i}$, which separate $R_{i}$ from $R_{j}$ and $R_{k}$ respectively (see figure), with possibly $j=k$. Suppose that both edges have the same sign, which is the case if we have a homogeneous graph. Then $e \in E_{i j} \Longleftrightarrow f \in E_{k i}$.

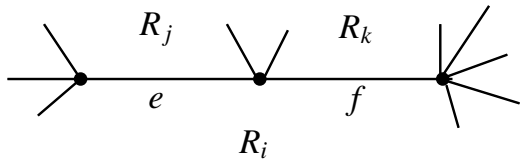

Remark 8. For a homogeneous block with sign $\epsilon$, the sum of two transposed elements in the corresponding Seifert matrix gives

$$
\operatorname{lk}\left(g_{i}, g_{j}^{+}\right)+\operatorname{lk}\left(g_{j}, g_{i}^{+}\right)=\epsilon\left|E_{i j}\right|+\epsilon\left|E_{j i}\right|=\epsilon\left|\partial R_{i} \cap \partial R_{j}\right| .
$$

The directed dual graph. A description of the Seifert matrix corresponding to a homogeneous block can be better understood as a certain matrix of degrees for the oriented dual graph. To construct the directed dual graph we draw a vertex $v_{i}$ in the region $R_{i}$, including a vertex $v_{r+1}$ for the unbounded region $R_{r+1}$, and for each edge $e$ in $\partial R_{i} \cap \partial R_{j}$ we draw an edge $\bar{e}$ joining $v_{i}$ and $v_{j}$, the edge $\bar{e}$ intersecting the original graph only in $e$. Moreover, the edge $\bar{e}$ is oriented from $v_{i}$ to $v_{j}$ if (and only if) $e \in E_{i j}$. The directed dual graph in the case of our running example is exhibited in the figure, assuming the sign $\epsilon=+1$ for all the edges and for the top left vertex.

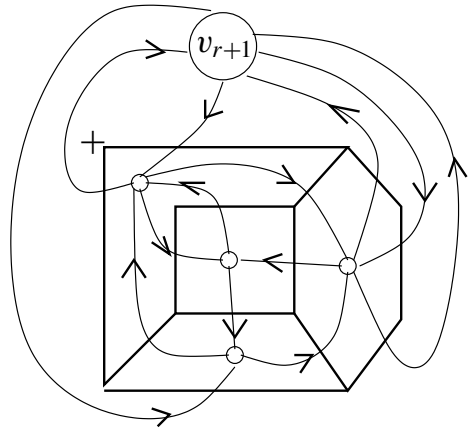

Note that the edges incident at any vertex have alternative orientations, which is equivalent to the second property of the sets $E_{i j}$ 's. In particular the degrees of the vertices are even numbers.

We now define $m_{i i}=-\epsilon \operatorname{deg}_{i}$ and $m_{i j}=\epsilon \operatorname{deg}_{i j}$, where $\operatorname{deg}_{i}$ is the number of edges leaving (or going to) $v_{i}$ and $\operatorname{deg}_{i j}$ is the number of edges from $v_{i}$ to $v_{j}$. It turns out that the matrix $\left(m_{i j}\right)_{1 \leq i, j \leq r+1}$ has determinant zero, and we obtain the Seifert matrix of the block by just deleting its last row and column. In our running 
example, for $\epsilon=+1$, we would have

$$
\left(\begin{array}{rrrrr}
-3 & 1 & 1 & 0 & 1 \\
1 & -2 & 0 & 1 & 0 \\
0 & 1 & -3 & 0 & 2 \\
1 & 0 & 1 & -2 & 0 \\
1 & 0 & 1 & 1 & -3
\end{array}\right) .
$$

One should note that this is essentially what Proposition 13.21 in [Burde and Zieschang 2003] states, where the adjective special is applied to a diagram if the union of the black regions (assuming a chessboard coloring in which the unbounded region is white) is the image of a Seifert surface under the projection that defines the diagram.

Properties of the matrix for a homogeneous block. Sard matrices. Let $\epsilon$ be a sign, +1 or -1 . A square matrix $A$ is said to be $\epsilon$-signed if its diagonal elements have sign $-\epsilon$ (in particular they do not vanish) and the elements out of the diagonal are zero or have sign $\epsilon$. The matrix $A$ is said to be row-dominant if for any row $i$ we have $\left|a_{i i}\right| \geq \sum_{j \neq i}\left|a_{i j}\right|$. The matrix $A$ is said to be strictly ascending row-dominant (abbreviated, sard) if $A$ is row-dominant and, in addition, there is an order of its rows $i_{1}<\cdots<i_{r}$ such that $\left|a_{i_{r} i_{r}}\right|>0$ and for any $k \in\{1, \ldots, r-1\}$ we have that $\left|a_{i_{k} i_{k}}\right|>\sum_{j \neq i_{1}, i_{2}, \ldots, i_{k}}\left|a_{i_{k} j}\right|$.

The following matrix can be seen to be $(+)$-signed and sard choosing the order $3,1,2$ for its rows (note that the condition $\left|a_{i_{r} i_{r}}\right|>0$ is for sure if $A$ is $\epsilon$-signed):

$$
\left(\begin{array}{rrr}
-3 & 0 & 3 \\
0 & -2 & 1 \\
1 & 0 & -2
\end{array}\right)
$$

Theorem 9. Let $B$ be a homogeneous block with sign $\epsilon$. Then there exists a basis of $H_{1}(B)$ such that the associated Seifert matrix $M$ is $\epsilon$-signed and sard.

Proof. Consider the basis of $H_{1}(B)$ given by the counterclockwise oriented cycles $\left\{g_{1}, \ldots, g_{r}\right\}$, boundaries of the bounded regions $R_{i}$ of $B$. Then the Seifert matrix $M=\left(a_{i j}\right)_{1 \leq i, j \leq r}$ is obviously $\epsilon$-signed since $a_{i i}=\operatorname{lk}\left(g_{i}, g_{i}^{+}\right)=-\epsilon k_{i}$ where $k_{i}$ is half the number of edges in the boundary of $R_{i}$, and $a_{i j}=\operatorname{lk}\left(g_{i}, g_{j}^{+}\right)=\epsilon\left|E_{i j}\right|$ if $i \neq j$. To see that $A$ is row-dominant note that $\left|a_{i i}\right|=k_{i}$, and on the other hand $\sum_{j \neq i}\left|a_{i j}\right|=\sum_{j \neq i}\left|E_{i j}\right| \leq k_{i}$, the inequality by the second property of the sets $E_{i j}$. We finally check that the matrix $M$ is sard, by finding an order $i_{1}<\cdots<i_{r}$ for its rows such that $\left|a_{i_{k} i_{k}}\right|>\sum_{j \neq i_{1}, i_{2}, \ldots, i_{k}}\left|a_{i_{k} j}\right|$ for any $k \in\{1, \ldots, r-1\}$. By the second property of the sets $E_{i j}$ there is always a bounded region $R_{i}$ such that $E_{i, r+1} \neq \varnothing$. The corresponding row is chosen to be the first one in this order, that is, $i_{1}=i$. Note that, since $\left|a_{i i}\right| \geq \sum_{1 \leq j \leq r+1, j \neq i}\left|E_{i j}\right|$ and $E_{i, r+1} \neq \varnothing$, it 
follows that $\left|a_{i i}\right|>\sum_{j \neq i}\left|a_{i j}\right|$. Now, when we delete the $i$-th row and column, the remaining matrix corresponds to the graph that remains after deleting the region $R_{i}$ (precisely, deleting the intersection between $R_{i}$ and $R_{r+1}$ ). The region can be also taken in such a way that the remaining graph is still a homogeneous block, hence the repetition of this process provides the wanted order for the rows of $M$.

The determinant for a homogeneous block. In this section we will prove that, given a block with sign $\epsilon$ and rank $r$, the determinant of the corresponding submatrix is nonzero, and its sign is equal to $(-\epsilon)^{r}$. To see this we just need a purely algebraic result due to Murasugi. For the convenience of the reader, we reproduce here its proof in a slightly different way:

Theorem 10 [Murasugi 1960, Section 2]. Let A be a square matrix of order $r$, $\epsilon$-signed and sard. Then $\operatorname{det} A<0$ if $\epsilon=+1$ and $r$ is odd, and $\operatorname{det} A>0$ otherwise. In other words, $\operatorname{det} A$ does not vanish and has sign $(-\epsilon)^{r}$.

Proof. By induction on $r$. The case $r=1$ (odd) is trivial; for $A=(a)$ we have $\operatorname{det} A=a$, and the result follows from the fact that $A$ is $\epsilon$-signed.

Now assume the statement for cases 1 to $r-1$, and consider the case $r$. Since $A$ is sard, there is an order $i_{1}<\cdots<i_{r}$ of the rows such that for any $k \in\{1, \ldots, r-1\}$ we have $\left|a_{i_{k} i_{k}}\right|>\sum_{j \neq i_{1}, i_{2}, \ldots, i_{k}}\left|a_{i_{k} j}\right|$. In particular, we have

$$
a_{i_{1} i_{1}}=-\sum_{j \neq i_{1}} a_{i_{1} j}-\lambda
$$

with $\lambda \neq 0$ and sign $\epsilon$. We now develop the determinant by the $i_{1}$-row, obtaining

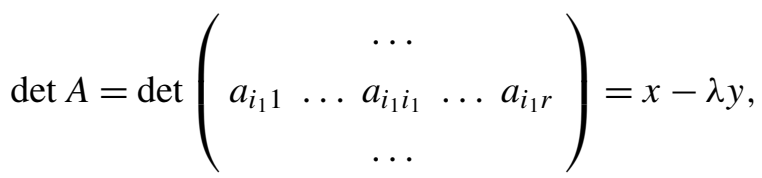

where

$$
x=\operatorname{det}\left(\begin{array}{c}
\ldots \\
a_{i_{1} 1} \ldots-\sum_{j \neq i_{1}} a_{i_{1} j} \ldots a_{i_{1} r} \\
\ldots
\end{array}\right)
$$

and $y$ is the determinant of the square matrix of order $r-1$, obtained by deleting the $i_{1}$-th row and column. Since this matrix is also $\epsilon$-signed and sard, by induction $y=(-\epsilon)^{r-1}|y| \neq 0$. Moreover, if each $a_{i_{1} j}=0$ for $j \neq i_{1}$ then $x=0$ obviously; otherwise it is a square matrix of order $r, \epsilon$-signed and row-dominant, and by Lemma 11, either $x=0$ or $x$ has sign $(-\epsilon)^{r}$. Then

$$
\operatorname{det} A=x-\lambda y=(-\epsilon)^{r}|x|-\epsilon|\lambda|(-\epsilon)^{r-1}|y|=(-\epsilon)^{r}(|x|+|\lambda||y|)
$$

and the result follows since $|\lambda|>0,|y|>0$ and $|x| \geq 0$. 
Lemma 11. Let $A$ be a square matrix of order $r, \epsilon$-signed and row-dominant. Then $\operatorname{det} A \leq 0$ if $\epsilon=+1$ and $r$ is odd, and $\operatorname{det} A \geq 0$ otherwise.

Proof. For technical reasons in the induction argument, we will prove this result for a slightly wider category of matrices, the weak $\epsilon$-signed and row-dominant matrices. For this matrices the condition of being $\epsilon$-signed is relaxed for allowing zeros in the diagonal.

We proceed by induction on $r$. The case $r=1$ is trivial. Assume now the statement for cases 1 to $r-1$, and consider the case $r$. Since $A$ is weak $\epsilon$-signed and row-dominant, each diagonal element of $A$ can be written as $a_{i i}=-\sum_{j \neq i} a_{i j}-\lambda_{i}$ with $\lambda_{i}=0$ or with sign $\epsilon$.

Let $A_{i}$ be the same matrix as $A$ except for possibly the first $i$ elements of its diagonal, where $a_{i i}$ is replaced by $a_{i i}+\lambda_{i}$. Let $A_{0}=A$. It turns out that

$$
\operatorname{det} A=\operatorname{det} A_{r}-\sum_{i=1}^{r} \lambda_{i} \operatorname{det}\left(\left(A_{i-1}\right)_{i}^{i}\right) \text {, }
$$

where the notation $B_{i}^{i}$ is used to denote the matrix obtained from $B$ by deleting its $i$-th row and $i$-th column. This follows from the fact that, for $k=1, \ldots, r$,

$$
\operatorname{det} A_{k-1}=\operatorname{det} A_{k}-\lambda_{k} \operatorname{det}\left(\left(A_{k-1}\right)_{k}^{k}\right) .
$$

Note that the determinant of $A_{r}$ is equal to zero, since the sum of all the elements of each row is zero. Moreover, each matrix $\left(A_{i-1}\right)_{i}^{i}$ is also weak $\epsilon$-signed and rowdominant, and has order $r-1$. By induction, its determinant is zero or has sign $(-\epsilon)^{r-1}$. Since each $\lambda_{i}$ is zero or has sign $-\epsilon$, the result follows.

Here is an application of the argument developed in this section:

Claim. Let $L$ be an oriented link which has a special alternating diagram. Then the leading coefficient of $\nabla_{L}(z)$ is \pm 1 if and only if $L$ is the connected sum of $(2, q)$-torus links.

Proof. Assume that $L$ is the connected sum of $(2, q)$-torus links. Since $\nabla_{L \sharp L^{\prime}}(z)=$ $\nabla_{L}(z) \nabla_{L^{\prime}}(z)$, it is enough to show that the leading coefficient of $\nabla_{L}(z)$ is \pm 1 if $L$ is a $(2, q)$-torus link. The diagram of $L$ is then of this form, or its mirror image:

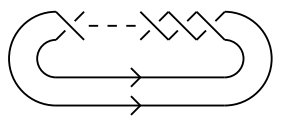

It has $q$ crossings, all with the same sign $\epsilon$. The corresponding Seifert graph, shown on the right, is a homogeneous block $B$ with two vertices and $q$ edges, all of them with $\operatorname{sign} \epsilon$.

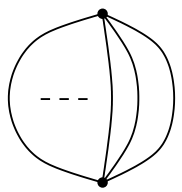

Following the process explained at the beginning of this section, we obtain the Seifert matrix $M=\left(m_{i, j}\right)_{i, j=1, \ldots, q-1}$ where $m_{i, i}=-\epsilon$ for $i=1, \ldots, q-1$, 
$m_{i, i+1}=\epsilon$ for $i=1, \ldots, q-2$, and $m_{i, j}=0$ otherwise. Then the leading coefficient of $\nabla_{L}(z)$ is $\epsilon^{\mathrm{rk} B} \mid$ det $M \mid=\epsilon^{q-1}$ since rk $B=1-v+e=1-2+q=q-1$.

Suppose now that the leading coefficient of $\nabla_{L}(z)$ is \pm 1 , and $L$ has a special alternating diagram $D$. Then $D$ is the connected sum of diagrams $D_{1}, \ldots, D_{r}$, where each $D_{i}$ is a diagram (of a link $L_{i}$ ) such that its Seifert graph has only one (homogeneous) block:

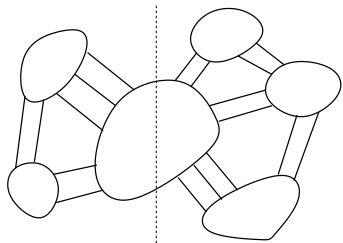

Clearly, $L=\sharp_{i=1}^{r} L_{i}$. Since $\nabla_{L}(z)=\prod_{i=1}^{r} \nabla_{L_{i}}(z)$ and $\nabla_{L}(z) \in Z\left[z^{ \pm 1}\right]$, the leading coefficient of each $\nabla_{L_{i}}(z)$ is \pm 1 . Hence it is enough to prove that $L$ is a $(2, q)$-torus link assuming that the leading coefficient of $\nabla_{L}(z)$ is \pm 1 , and $L$ has a diagram $D$ whose associated Seifert graph is a homogeneous block $B$, let's say with sign $\epsilon$.

We will prove that $B$ has the desired form (reproduced on the right for convenience). We do this by induction on the number of edges of $B$. With this aim, we order the $r$ bounded regions of $B$ as in the proof of Theorem 9. The corresponding Seifert matrix $A$ is then $\epsilon$-signed and sard, and by the proof of Theorem 10, we have

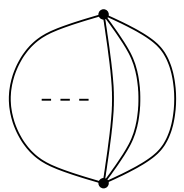

$$
\operatorname{det} A=(-\epsilon)^{r}(|x|+|\lambda||y|),
$$

where $y=\operatorname{det} A_{1}^{1} \neq 0$. Since the leading coefficient of $\nabla_{L}(z)$ is \pm 1 , we have $\operatorname{det} A= \pm 1$; since $\lambda$ and $y$ are nonzero integers, we have $y=\operatorname{det} A_{1}^{1}= \pm 1$.

Now, according to the proof of Theorem $9, A_{1}^{1}$ is the Seifert matrix associated to the diagram $D^{\prime}$ whose Seifert graph is $B^{\prime}=B \backslash\left(R_{1} \cap R_{r+1}\right)$, where $R_{r+1}$ is the unbounded region of $B$. Since $B^{\prime}$ is still a homogeneous block, by induction we have that $B^{\prime}$ has the form shown above and to the right, and $B$ adds a path connecting the two vertices of $B^{\prime}$ in the unbounded region of $B^{\prime}$ :

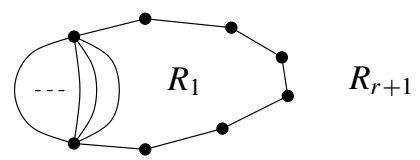

Let $2 k$ be the number of edges bounding $R_{1}$ in $B$. Then the original Seifert matrix is

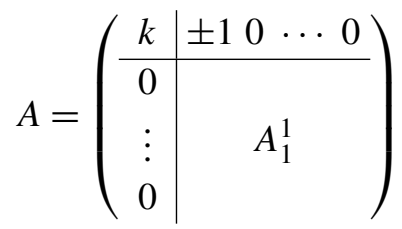


or its transpose, in any case with determinant $\pm k$. Hence $k=1$ and the result follows.

Corollary 12. Let $L$ be an oriented homogeneous link. Then the leading coefficient of $\nabla_{L}(z)$ equals \pm 1 if and only if $L$ is the Murasugi sum of connected sums of $(2, q)$-torus links.

\section{Homogeneous knots of genus one}

We finish the paper with a complete classification of the family of homogeneous knots of genus one. Let $D$ be a homogeneous diagram of a homogeneous knot $K$ of genus one. Let $F$ and $G$ be respectively the projection surface and the Seifert graph associated to the diagram $D$. We already know that the genus of $F$ is exactly the genus of the knot. Since $2 g(F)+\mu-1=\operatorname{rk} G$ and $K$ is a link with one component, we deduce that $G$ has rank two. Here are the two types of graphs:
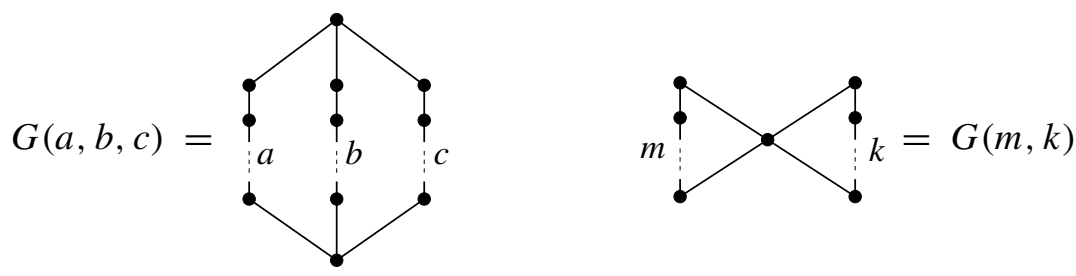

Homogeneous graphs with rank two: one and two blocks

As indicated, we name them $G(a, b, c)$ and $G(m, k)$, respectively; the absolute values of the integers $a, b, c, m, k$ are the numbers of corresponding edges, and their signs are the signs of these edges.

Note that these graphs could have some tails, but this would not affect to the knot type. Since $G(a, b, c)$ is homogeneous and has only one block, $a, b, c$ must have all the same sign; since $F$ is orientable, they have also the same parity. On the contrary, $G(m, k)$ has two blocks, hence $m$ and $k$ can have different signs, but both must be even because of the orientability. Note also that the second graph can be considered a degenerated form of the first one, with $b=0$.

In general, the Seifert graph does not determine the link where it comes from, although in the first case it does. In $G(a, b, c)$ there are exactly two trivalent vertices; the corresponding Seifert circles can be one inside the other, or separated. When viewed this in the sphere $S^{2}$ there is no difference, and the corresponding knot is the pretzel knot with diagram $P(a, b, c)$. Moreover, since $P(a, b, c)$ must be a knot, the numbers $a, b, c$ should be all odd, or exactly one of them should be even. It follows that all of them are odd.

Now consider the graph with two blocks, $G(m, k)$. There is only one vertex with valence four, given the two possible configurations for the Seifert circles shown in 
the figure (which illustrates the case $|m|=|k|=4$ ):
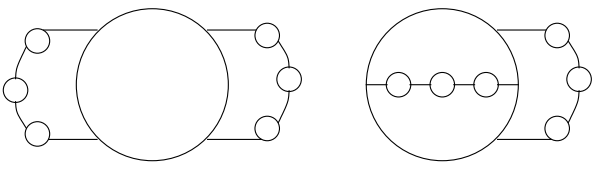

The first configuration corresponds to a link with three components, and the second corresponds to a knot $K$ (see figure below). Moreover, the knot $K$ obtained is also a pretzel knot, given by the pretzel diagram $D(m, k)=P\left(m, \epsilon, \mid{ }^{|k|} ., \epsilon\right)$, where $m$ and $k$ are even integers and $\epsilon$ is the sign of $k$. For example, $D(4,-2)=$ $P(4,-1,-1)$ is the example in the right diagram:
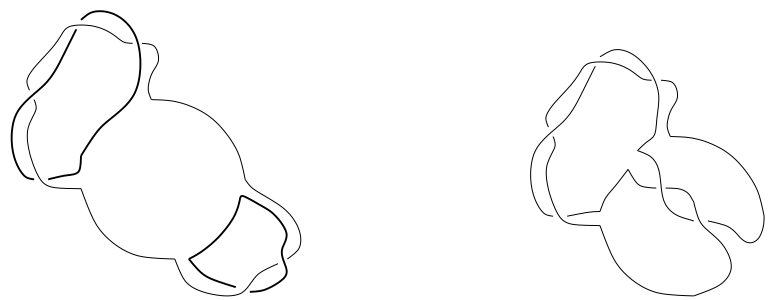

What we have done is to prove the following result:

Theorem 13. A genus-one knot is homogeneous if and only if it belongs to one of the two following classes of knots:

(1) Pretzel knots with diagram $P(a, b, c)$, where $a, b, c$ are odd integers with the same sign.

(2) Pretzel knots with diagram $D(m, k)=P(m, \epsilon,|k|, \epsilon)$, where $m$ and $k$ are nonzero even integers and $\epsilon=k /|k|$ is the sign of $k$.

This classification and some partial information from the Jones polynomial allow us to give another proof of the following result:

Corollary 14 [Cromwell 1989]. Pretzel knots $P(p,-q,-r)$ with $3 \leq p \leq q \leq r$, all of them odd, are not homogeneous.

In the original proof, Cromwell calculated the Homfly polynomial $P(v, z)=$ $\sum_{i=0}^{r} \alpha_{i}(v) z^{i}$ and checked that $\alpha_{r}(v)$ contains terms of both signs [Cromwell 1989, Theorem 10]. But, for homogeneous links, these coefficients are all nonnegative or all nonpositive, according to a result [ibid, Corollary 4.3] due to Traczyk.

Proof. We want to prove that the knot $K$ defined by a pretzel diagram $P(p,-q,-r)$ is not homogeneous. First note that $K$ has genus one, since the projection surface defined by the diagram $P(p,-q,-r)$ has Euler characteristic -1 , hence genus one, and $K$ is not the trivial knot; for example, according to [Manchón 2003, Theorem 2, case (iv)(a)], the span of its Jones polynomial (with normalization 
$\left.-t^{-1 / 2}-t^{1 / 2}\right)$ is $p+q+r-\min \{p, q-1\}$, which is different from one since $3 \leq p \leq q, r$.

Now, the lowest degree and the coefficient of the highest degree term of the Jones polynomial tell us that $K$ does not belong to any of the two classes of homogeneous knots of genus one given by Theorem 13, as the following table shows:

\begin{tabular}{|c|c|c|c|}
\hline \multicolumn{2}{|c|}{ Knot diagram } & Lowest degree & $\begin{array}{c}\text { Coefficient of the } \\
\text { highest degree term }\end{array}$ \\
\hline \multirow{2}{*}{$P(p,-q,-r)$} & $3 \leq p<q \leq r$ & $1 / 2$ & -1 \\
\cline { 2 - 4 } & $3 \leq p=q \leq r$ & $-1 / 2$ & \\
\hline \multirow{2}{*}{$P(a, b, c)$} & $0 \leq a, b, c$ & $-3 / 2-a-b-c$ & \\
\cline { 2 - 4 } & $a, b, c \leq 0$ & $1 / 2$ & 1 \\
\hline \multirow{3}{*}{$D(m, k)$} & $m, k>0$ & $-m-1 / 2$ & \\
\cline { 2 - 4 } & $m<0, k>0$ & $1 / 2$ & \\
\cline { 2 - 4 } & $m>0, k<0$ & $k-m-1 / 2$ & \\
\cline { 2 - 4 } & $m, k<0$ & $k-1 / 2$ & \\
\hline
\end{tabular}

Note that the Conway polynomial together with the span of the Jones polynomial are not enough in order to prove Corollary 14. According to the values displayed in the following table, we have for example that the knots defined by the diagrams $P(3,-45,-91)$ and $P(11,23,101)$ share Conway polynomial and the span of their Jones polynomials, and the same happens to the pair of knots defined by the diagrams $P(11,-15,-15)$ and $D(-4,26)$.

\begin{tabular}{|c|c|c|c|}
\hline \multicolumn{2}{|c|}{ Knot diagram } & Conway polynomial & Jones polynomial span \\
\hline \multirow{2}{*}{$P(p,-q,-r)$} & $3 \leq p<q \leq r$ & \multirow{2}{*}{$(q r-p q-p r+1) / 4$} & $q+r$ \\
\hline & $3 \leq p=q \leq r$ & & $q+r+1$ \\
\hline \multirow{2}{*}{$P(a, b, c)$} & $0 \leq a, b, c$ & \multirow{2}{*}{$(a b+a c+b c+1) / 4$} & $1+a+b+c$ \\
\hline & $a, b, c \leq 0$ & & $1-a-b-c$ \\
\hline \multirow{4}{*}{$D(m, k)$} & $m, k>0$ & \multirow{4}{*}{$m k / 4$} & $1+m+k$ \\
\hline & $m<0, k>0$ & & $k-m$ \\
\hline & $m>0, k<0$ & & $m-k$ \\
\hline & $m, k<0$ & & $1-m-k$ \\
\hline
\end{tabular}


We also have the following result (as above, the Jones polynomial of the pretzel links and their spans have been calculated following [Manchón 2003]):

Corollary 15. At least one of the extreme coefficients of the Jones polynomial of a homogeneous knot of genus one is -1 .

Finally, we remark that Stoimenow [2008] has showed that a genus-two homogeneous knot is alternating or positive. Jong and Kishimoto [2009] have studied genus-two positive knots extensively.

\section{Acknowledgments}

I am grateful to Prof. Hugh R. Morton for several helpful comments on a previous version of this paper. I am also grateful to the referee for a number of helpful suggestions for improving the article. An overview of this work was presented at the Conference Knots in Poland III; I am grateful to its organizers for their hospitality.

\section{References}

[Abe 2011] T. Abe, “The Rasmussen invariant of a homogeneous knot", Proc. Amer. Math. Soc. 139:7 (2011), 2647-2656. MR 2012c:57010 Zbl 1226.57004

[Burde and Zieschang 2003] G. Burde and H. Zieschang, Knots, 2nd ed., De Gruyter Studies in Mathematics 5, De Gruyter, Berlin, 2003. MR 2003m:57005 Zbl 1009.57003

[Cromwell 1989] P. R. Cromwell, "Homogeneous links", J. London Math. Soc. (2) 39:3 (1989), 535-552. MR 90f:57001 Zbl 0685.57004

[Cromwell 2004] P. R. Cromwell, Knots and links, Cambridge University Press, Cambridge, 2004. MR 2005k:57011 Zbl 1066.57007

[Crowell 1959] R. H. Crowell, "Genus of alternating link types”, Ann. of Math. (2) 69:2 (1959), 258-275. MR 20 \#6103b Zbl 0111.35803

[Diestel 2005] R. Diestel, Graph theory, 3rd ed., Grad. Texts in Math. 173, Springer, Berlin, 2005. MR 2006e:05001 Zbl 1093.05001

[Gabai 1983] D. Gabai, "The Murasugi sum is a natural geometric operation", pp. 131-143 in Low dimensional topology (San Francisco, 1981), edited by S. J. Lomonaco, Jr., Contemp. Math. 20, Amer. Math. Soc., Providence, RI, 1983. MR 85d:57003 Zbl 0524.57004

[Jong and Kishimoto 2009] I. D. Jong and K. Kishimoto, "On positive knots of genus two", preprint, Osaka City University, 2009, available at http://jong.web.fc2.com/posi.pdf.

[Manchón 2003] P. M. G. Manchón, "On the Kauffman bracket of pretzel links", Marie Curie Fellowships Annals 2 (2003), 118-122.

[Melvin and Morton 1986] P. M. Melvin and H. R. Morton, "Fibred knots of genus 2 formed by plumbing Hopf bands", J. London Math. Soc. (2) 34:1 (1986), 159-168. MR 87m:57006 Zbl 0576.57008

[Murasugi 1958a] K. Murasugi, "On the genus of the alternating knot, I", J. Math. Soc. Japan 10:1 (1958), 94-105. MR 20 \#6103a Zbl 0084.19301

[Murasugi 1958b] K. Murasugi, “On the genus of the alternating knot, II”, J. Math. Soc. Japan 10:3 (1958), 235-248. MR 20 \#6103a Zbl 0106.16701 
[Murasugi 1960] K. Murasugi, “On alternating knots”, Osaka Math. J. 12:2 (1960), 277-303. MR 25 \#563 Zbl 0113.38603

[Ozawa 2011] M. Ozawa, "Essential state surfaces for knots and links", J. Austral. Math. Soc. 391 (2011), 365-390.

[Stallings 1978] J. R. Stallings, "Constructions of fibred knots and links", pp. 55-60 in Algebraic and geometric topology, II (Stanford, CA, 1976), edited by R. J. Milgram, Proc. Sympos. Pure Math. 32, Amer. Math. Soc., Providence, RI, 1978. MR 80e:57004 Zbl 0394.57007

[Stoimenow 2008] A. Stoimenow, "Knots of (canonical) genus two", Fund. Math. 200:1 (2008), 1-67. MR 2009m:57015 Zbl 1190.57008

Received February 16, 2011. Revised August 29, 2011.

PEDRo M. GonZÁlez MANCHÓN

DEPARTMENT OF APPLIED MATHEMATICS

EUITI - UNIVERSIDAD POLITÉCNICA DE MADRID

RONDA DE VALENCIA 3

28012 MADRID

SPAIN

pedro.gmanchon@upm.es

http://gestion.euiti.upm.es/index/departamentos/matematicas/manchon/index.htm 


\title{
PACIFIC JOURNAL OF MATHEMATICS
}

\author{
http://pacificmath.org \\ Founded in 1951 by \\ E. F. Beckenbach (1906-1982) and F. Wolf (1904-1989)
}

\section{EDITORS}

V. S. Varadarajan (Managing Editor)

Department of Mathematics

University of California

Los Angeles, CA 90095-1555

pacific@math.ucla.edu

Vyjayanthi Chari

Department of Mathematics

University of California

Riverside, CA 92521-0135

chari@math.ucr.edu

\section{Robert Finn}

Department of Mathematics Stanford University

Stanford, CA 94305-2125

finn@math.stanford.edu

Kefeng Liu

Department of Mathematics

University of California

Los Angeles, CA 90095-1555

liu@math.ucla.edu
Darren Long

Department of Mathematics

University of California

Santa Barbara, CA 93106-3080

long@math.ucsb.edu

Jiang-Hua Lu

Department of Mathematics

The University of Hong Kong

Pokfulam Rd., Hong Kong jhlu@maths.hku.hk

Alexander Merkurjev

Department of Mathematics

University of California

Los Angeles, CA 90095-1555

merkurev@math.ucla.edu
Sorin Popa

Department of Mathematics University of California

Los Angeles, CA 90095-1555 popa@math.ucla.edu

Jie Qing

Department of Mathematics

University of California

Santa Cruz, CA 95064

qing@cats.ucsc.edu

Jonathan Rogawski

Department of Mathematics

University of California

Los Angeles, CA 90095-1555

jonr@math.ucla.edu

\section{PRODUCTION}

pacific@math.berkeley.edu

\section{SUPPORTING INSTITUTIONS}

ACADEMIA SINICA, TAIPEI

CALIFORNIA INST. OF TECHNOLOGY INST. DE MATEMÁTICA PURA E APLICADA KEIO UNIVERSITY

MATH. SCIENCES RESEARCH INSTITUTE NEW MEXICO STATE UNIV.

OREGON STATE UNIV.

\author{
STANFORD UNIVERSITY \\ UNIV. OF BRITISH COLUMBIA \\ UNIV. OF CALIFORNIA, BERKELEY \\ UNIV. OF CALIFORNIA, DAVIS \\ UNIV. OF CALIFORNIA, LOS ANGELES \\ UNIV. OF CALIFORNIA, RIVERSIDE \\ UNIV. OF CALIFORNIA, SAN DIEGO \\ UNIV. OF CALIF., SANTA BARBARA
}

\author{
UNIV. OF CALIF., SANTA CRUZ \\ UNIV. OF MONTANA \\ UNIV. OF OREGON \\ UNIV. OF SOUTHERN CALIFORNIA \\ UNIV. OF UTAH \\ UNIV. OF WASHINGTON \\ WASHINGTON STATE UNIVERSITY
}

These supporting institutions contribute to the cost of publication of this Journal, but they are not owners or publishers and have no responsibility for its contents or policies.

See inside back cover or pacificmath.org for submission instructions.

The subscription price for 2012 is US \$420/year for the electronic version, and \$485/year for print and electronic.

Subscriptions, requests for back issues from the last three years and changes of subscribers address should be sent to Pacific Journal of Mathematics, P.O. Box 4163, Berkeley, CA 94704-0163, U.S.A. Prior back issues are obtainable from Periodicals Service Company, 11 Main Street, Germantown, NY 12526-5635. The Pacific Journal of Mathematics is indexed by Mathematical Reviews, Zentralblatt MATH, PASCAL CNRS Index, Referativnyi Zhurnal, Current Mathematical Publications and the Science Citation Index.

The Pacific Journal of Mathematics (ISSN 0030-8730) at the University of California, c/o Department of Mathematics, 969 Evans Hall, Berkeley, CA 94720-3840, is published monthly except July and August. Periodical rate postage paid at Berkeley, CA 94704, and additional mailing offices. POSTMASTER: send address changes to Pacific Journal of Mathematics, P.O. Box 4163, Berkeley, CA 94704-0163.

PJM peer review and production are managed by EditFLOW ${ }^{\mathrm{TM}}$ from Mathematical Sciences Publishers.

PUBLISHED BY PACIFIC JOURNAL OF MATHEMATICS

at the University of California, Berkeley 94720-3840

A NON-PROFIT CORPORATION

Typeset in LATEX

Copyright $(02012$ by Pacific Journal of Mathematics 


\section{PACIFIC JOURNAL OF MATHEMATICS}

Volume $255 \quad$ No. $2 \quad$ February 2012

On the local Langlands correspondences of DeBacker-Reeder and

Reeder for $\operatorname{GL}(\ell, F)$, where $\ell$ is prime

MOSHE ADRIAN

$R$-groups and parameters

281

DUBRAVKA BAN and DAVID GOLDBERG

Finite-volume complex-hyperbolic surfaces, their toroidal

305

compactifications, and geometric applications

LUCA FABRIZIO Di CERBO

Character analogues of Ramanujan-type integrals involving the Riemann 317 $\Xi$-function

ATUl DiXIT

Spectral theory for linear relations via linear operators

DANA GHEORGHE and FLORIAN-HORIA VASILESCU

Homogeneous links and the Seifert matrix

PEDRO M. GonZÁlez MANCHÓN

Quantum affine algebras, canonical bases, and $q$-deformation of arithmetical functions

HENRY H. KIM and KYU-HWAN LEE

Dirichlet-Ford domains and arithmetic reflection groups

GRANT S. LAKELAND

Formal equivalence of Poisson structures around Poisson submanifolds

IOAN MĂRCUT,

A regularity theorem for graphic spacelike mean curvature flows

BENJAMIN STUART THORPE

Analogues of level- $N$ Eisenstein series 